\title{
Hand-Held Steerable Needle Device
}

\author{
R. Ebrahimi, S. Okazawa, R. Rohling, and S.E. Salcudean \\ Department of Electrical and Computer Engineering \\ University of British Columbia, Vancouver, Canada \\ \{rohling, tims\}@ece.ubc.ca
}

\begin{abstract}
This paper presents a novel handheld device for steerable needle insertions for minimally invasive percutaneous procedures. Controlled lateral deflection of over $30 \mathrm{~mm}$ during a $100 \mathrm{~mm}$ axial insertion is demonstrated with a 20 gauge needle. A handheld, motorized device has been built to control the direction and rate of steering according to a two-axis miniature joystick input. To facilitate interventions with this steerable needle, two methods of locating the needle and its tip using ultrasound imaging are presented. One uses needle tip vibration for colour/power Doppler-based localization, the other is based on realtime segmentation of B-mode images.
\end{abstract}

\section{Introduction}

Minimally invasive percutaneous procedures under ultrasound (US) guidance have a wide variety of applications in medical diagnoses and therapy. In these procedures, needle control presents a technical challenge for the physician. Assuming that the needle travels along a line, it must be perfectly aligned with the target prior to insertion. However, tissue inhomogeneity and tip bevel cause the needle to be deflected laterally during the insertion. To correct these deflection errors, limited steering may be achieved by manipulating the needle base 2 or the tip bevel direction [3. Several groups have implemented computer and robotic assistance to align the needle with the target [4|5|6/7/8|9] assuming known target location in 3D, patient immobilization, and a straight needle path.

US guidance presents further difficulties. The US image plane must be carefully aligned with the needle and the target to see the required trajectory. Speckle, shadows and other artifacts also inhibit the visualization of the needle in the image. Software algorithms that segment the needle from the rest of the ultrasound image have been the subject of recent research [1112. Alternatively, physical modification of the needle tip, such as scoring and coatings, can improve its ultrasound reflectivity [13].

The following research presents the first working components in a suite of proposed guidance and imaging solutions to the difficulties encountered in US-guided needle punctures. Based on the concepts presented in [1], a novel computer-controlled steerable needle device with a joystick for steering input has been built that takes the place of the conventional needles used in percutaneous procedures. The physician is able to produce immediate path corrections by 
directing the needle tip with the joystick, and curved access trajectories are possible. With this system, the current clinical procedure remains largely unchanged while the capabilities and error tolerance are expanded. Needle segmentation and tip localization techniques have also been developed to aid guidance.

\section{$2 \quad$ A Method for Needle Steering}

Although catheters often employ a steering mechanism at the tip, their designs are not easily adapted to needles because of the small diameter of the needles and the large forces involved in percutaneous procedures. Medical needles typically consist of a hollow tube called a cannula and a solid rod called a stylet which sits inside the cannula during insertion. Few steerable needles have been proposed previously [910]. In [9] a double cannula is constructed from two concentric prebent tubes. When the curvatures of the two tubes are aligned, the needle is bent. Rotating the inner tube with respect to the outer tube reduces the net bending force and straightens the needle. Steering is derived from rotation of the tubes by two motors housed in the handle. In [10] a telescoping double cannula and stylet are combined where only the inner cannula is curved and is made from a superelastic material such as nickel titanium. Steering is derived from rotation and extension of the inner cannula.

A new simpler needle steering concept, shown in Figure 1, is proposed here. This method employs a stylet that is longer than the cannula so that up to $2 \mathrm{~cm}$ of the stylet tip can be selectively exposed. The stylet has a mild curve over the exposed portion of the tip. During insertion, the needle steers in the direction of the exposed curve. Because of the geometry of their construction, the cannula is significantly stiffer than the stylet. Therefore, by withdrawing the stylet, the stiffer cannula straightens out the curve and the needle becomes approximately straight. The steering direction is selected by rotating the stylet and the steering rate is selected by extending the stylet and exposing its curve.

(a)

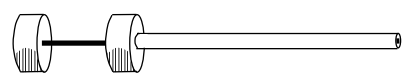

(b)

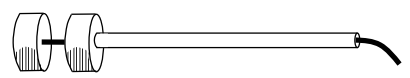

Fig. 1. Needle steering concept. (a) Stylet withdrawn. (b) Stylet exposed.

\section{Experimental Validation of Needle Steering}

The following experiment was designed to validate the effectiveness of the bentstylet steering concept in tissue phantoms. The stylet from a 20 gauge Chiba needle is manually given a $2 \mathrm{~mm}$ lateral curve opposite to the bevel direction over approximately a $15 \mathrm{~mm}$ range starting at the tip. The cannula and stylet 
are mounted to a horizontal linear slide, such that the tip curvature is in the horizontal plane. A $100 \mathrm{~mm}$ square phantom with a height of $15 \mathrm{~mm}$ made of a polyvinyl chloride compound is placed on a light table with a CCD camera mounted vertically overhead[2]. The camera view can be seen in Figure 2 (a). The needle is advanced by hand at a constant rate of $5 \mathrm{~mm} / \mathrm{s}$ into the phantom while the camera captures images of the insertion at 1 frame/s. Varying the insertion speeds within the range used in clinical practice (1 to $10 \mathrm{~mm} / \mathrm{s}$ ) had no effect on the trajectory produced by the tip curvature.

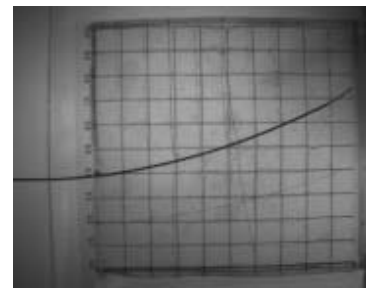

(a)

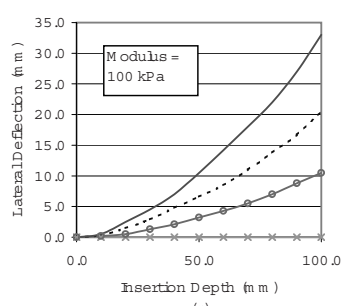

(c)

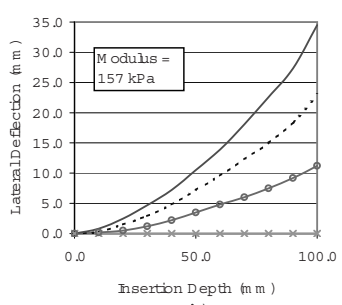

(b)

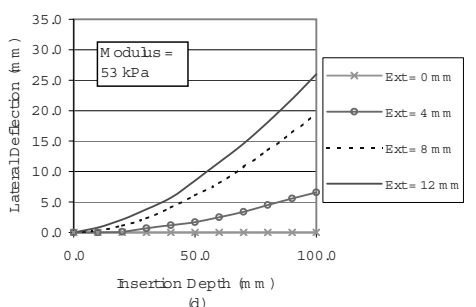

(d)

Fig. 2. Experimental results. (a) Lateral deflection of $34.5 \mathrm{~mm}$ for a $100 \mathrm{~mm}$ insertion with a $12 \mathrm{~mm}$ curve exposure in phantom 1 . Steering trajectory results using $2,4,8$, and $12 \mathrm{~mm}$ of curve exposure in (b) Phantom 1 (Young's Modulus $=157 \mathrm{kPa}$ ), (c) Phantom $2(100 \mathrm{kPa})$, and (d) Phantom $3(53 \mathrm{kPa})$.

Three tissue phantoms of different stiffnesses were created by varying the proportion of hardener used from $0 \%$ to $25 \%$ to $50 \%$. In each phantom, four variations on the amount of curve exposure were used: $2 \mathrm{~mm}, 4 \mathrm{~mm}, 8 \mathrm{~mm}$, and $12 \mathrm{~mm}$. The $2 \mathrm{~mm}$ extension trial is the base case where the steering effect of the slightly exposed curve exactly cancels that due to the needle bevel, thus causing the needle to travel straight.

As can be seen in Figure 2(b) through (d), varying the stylet extension produces a powerful and controllable steering effect. The steering rate decreases slightly with phantom compliance. Damage to tissue from needle steering is unlikely as long as the trajectory is kept smooth using conservative motions of the stylet. The risk of injury will be further examined in future studies. 


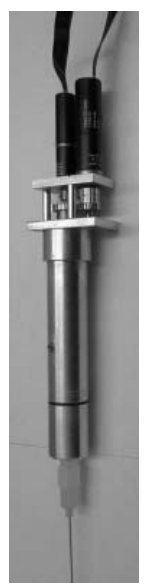

(a)

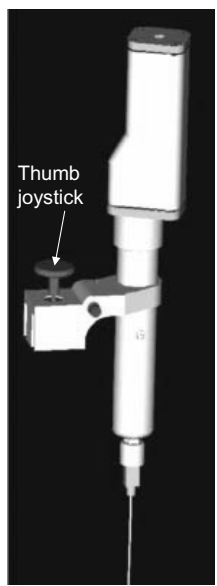

(b)

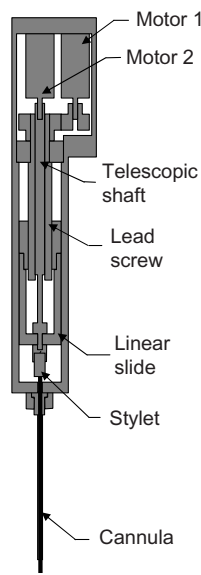

(c)

Fig. 3. Needle device. (a) Photograph, (b) Computer Rendering, and (c) Schematic.

\section{Electro-Mechanical Design}

The purpose of the needle steering device is to provide motorized actuation for the rotation and extension of the stylet with respect to the cannula. A photograph of the device, a rendering of the mechanical design and an internal schematic are shown in Figure [3. The device attaches to off-the-shelf, sterilizable medical needles using an ISO 594/1,2 international standard Luer-Lock connector. The cannula is rigidly fixed to the casing of the device. Internally, two DC motors drive mechanical components that generate the stylet extension and rotation. Figure 3 (b) shows a miniature thumb-joystick mounted on the side of the device for steering input, but other configurations are feasible including remote operation. The design achieves $20 \mathrm{~mm}$ of axial stylet motion and unlimited rotation in a compact package weighing approximately $200 \mathrm{~g}$ that can be fully operated with one hand.

As shown in Figure 3 (c), the extension of the stylet is driven by a leadscrew powered by motor 1 . The motor turns the leadscrew via two spurgears. The leadscrew nut is fixed to a linear slide that can translate along the axis of the needle. The stylet is mounted to this linear slide in a manner that allows it to rotate freely about its axis. Motor 2 directly drives the rotation of the stylet with a telescopic shaft. Each end piece of the telescopic shaft is free to slide with respect to the other along the shaft axis. The mating portion of the two pieces of the shaft is housed within a hole bored through the leadscrew.

The physician inputs steering commands using a miniature two-axis analog joystick. In the present design, shown in Figure 3 (b), the joystick is mounted on the shaft of the device facing opposite the insertion direction so that the physician can firmly hold the device in his or her palm and manipulate the joystick with the thumb. Position encoders on each motor and a quadrature counting circuit measure the absolute rotation and extension of the stylet. An HC11 microcontroller polls the joystick position (via A/D conversion), reads the 


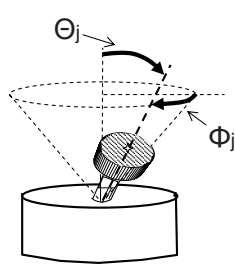

(a)

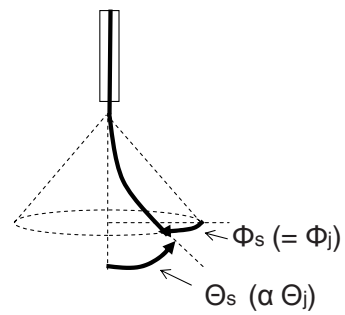

(b)

Fig. 4. Mapping of (a) joystick position to (b) stylet position.

encoder counters, and produces a PWM control signal for each motor. A motor current-drive circuit drives each motor based on the PWM signal.

The microcontroller runs two PID controllers in position feedback control to map the joystick position to a stylet rotation and extension. The mapping is shown in Figure 4. The movement of the joystick is described by $\Phi_{j}$ and $\Theta_{j}$ as shown. The rotation $\Phi_{j}$ maps directly to the stylet rotation $\Phi_{s}$ and controls the steering direction. The rotation $\Theta_{j}$ maps to the stylet extension. A greater extension produces a larger $\Theta_{s}$, therefore, $\Theta_{j}$ is proportional to $\Theta_{s}$ and controls the steering rate.

\section{Image Guided System Design}

Using the steerable needle device, the basic procedure for a percutaneous insertion would proceed as follows. The physician holds the device in one hand and an US transducer in the other while watching the US display. He or she orients the transducer so the imaging plane contains the target and the intended needle path. He or she then chooses an insertion point and orientation on the skin surface and proceeds to manually insert the needle along the desired path while watching the progress of the needle internally on the US display. Up to this point, the procedure is identical to current practice, but when deviations from the desired path occur they may be corrected by pushing the joystick in the direction toward the desired path and continuing the insertion. Early feedback from physicians suggest this implementation has immediate practical value in delicate biopsy, drug delivery and blood transfusion procedures.

The ability to steer the needle requires accurate knowledge of the current needle position. A needle segmentation algorithm automatically extracts this knowledge from the US images. This information can be used for semi or full automation of the basic freehand procedure and can augment visualization by displaying a clear needle overlay.

\subsection{Needle Segmentation Algorithm}

In its present form, the needle segmentation algorithm operates on static (nonreal-time) US images. Two initial points are input by the physician at the beginning of the procedure to indicate an approximate needle axis. 


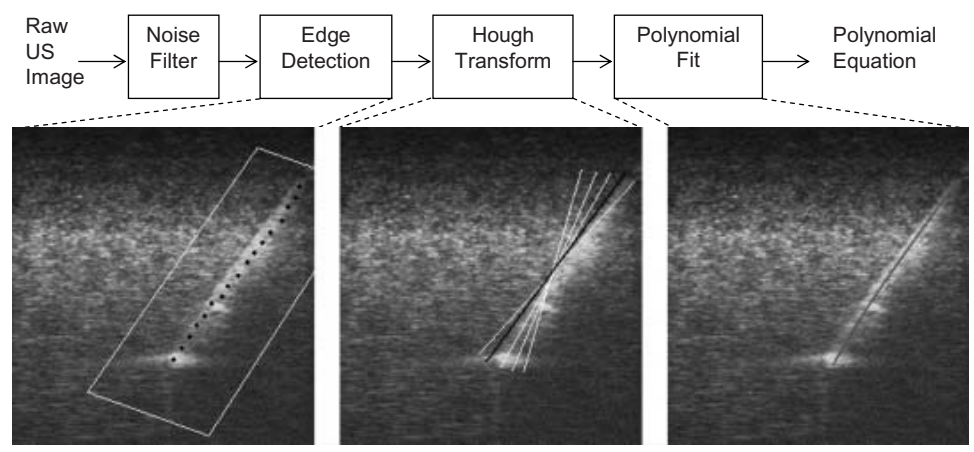

Fig. 5. Needle segmentation algorithm.

The algorithm consists of the following steps, shown in Figure 5 that act within a reduced region of each US image based on the estimated needle position. A noise filtering step uses a 10x10 median filter to remove the speckled texture of the US images. An edge detection step calculates linear derivatives along rays perpendicular to the estimated needle axis. Two large peaks are observed along each ray corresponding to the edges of the needle shaft. The mean position of each pair of peaks gives a point along each ray. A majority of these points lie on the needle. A Hough Transform is performed over an angular range limited to $+/-15^{0}$ from the estimated needle axis that identifies a line approximating the true needle axis. Outliers far from this line are rejected. The remaining points are fit to a polynomial equation which represents the curvature of the needle.

Although the direction of the needle can be estimated robustly, the tip is found with less accuracy because of the nature of the US image. In many cases, the presence of speckle and signal drop-out hinder the detection of the tip.

\subsection{Needle Tip Localization Using Stylet Vibration}

When the tip position is unclear, it is possible to improve detection by actively oscillating the stylet within the cannula [14]. The resulting oscillation of the nearby tissue can then be localized by the Doppler detection system found in most modern US scanners. In particular, power Doppler has been explored to localize the stylet tip while it is vibrated by the motors. In the HC11 software, a triangular wave with a frequency of $2 \mathrm{~Hz}$ and an amplitude of $2 \mathrm{~mm}$ is superimposed on the signal controlling stylet extension. This small oscillation does not affect the needle steering performance so it can be used during steering actions, but it is detectable using US power Doppler. The parameters of the Doppler measurement are adjusted to the stylet oscillatory frequency to isolate the tip location. Figure 6] demonstrates this technique in a tissue phantom.

\subsection{Advanced Implementation Options}

Using the steerable needle device and the needle segmentation and tip detection algorithms as fundamental components, a number of system variants are possible. The procedure described above is a freehand steerable needle technique with 


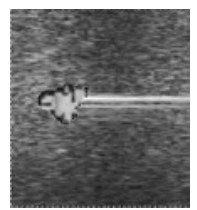

(a)

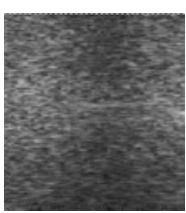

(b)

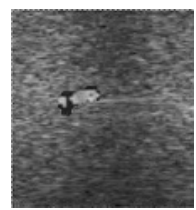

(c)

Fig. 6. (a) Needle in US plane with power Doppler. (b) Needle out of US plane without power Doppler. (c) Needle out of US plane with power Doppler.

US guidance and needle segmentation. Future implementations will use a physical tracking system to measure the positions of the needle device and the US transducer relative to a fixed base coordinate frame 1]. For example an Optotrack sensing system has been used to track US transducers by mounting LEDs to the transducer handle 15. This information will be used firstly by the needle segmentation algorithm to compute the estimated needle position in the US image. Secondly, by knowing the positions of points in the US image in absolute coordinates and the position of the steering device in the same coordinate frame, the computer software will be able to calculate the necessary steering commands and insertion speeds automatically so that either or both of these actions can be computer controlled. In these more advanced systems, the physician would simply define a desired target and path through a user interface.

\section{Conclusion}

The preceding research presents the design and implementation of the fundamental components in an image-guided steerable-needle system for use in percutaneous procedures. Experimental results show that the steering effect produced by the bent-stylet concept is very strong. In the stiffest phantom, over $30 \mathrm{~mm}$ of lateral deflection occurs during a $100 \mathrm{~mm}$ insertion with $12 \mathrm{~mm}$ of curve exposure. In the phantoms we considered, the steerability of the needle increases with tissue stiffness. A hand-held needle steering device was designed and built that produces the necessary stylet motions to achieve a desired steering rate and direction indicated by a joystick.

An US needle segmentation algorithm has been developed that is successful in segmenting the needle shaft from poor images with distracting bright features. For cases where the tip position is unclear, a Doppler-based tip detection technique using stylet vibration is shown to be an effective method for localizing the needle tip. A physical tracking system for the device and the US transducer will be implemented in future work. This will provide the needle segmentation algorithm with an estimate of the needle position and allows points in the US image to be known in absolute coordinates. For future versions of the system, automation of steering and insertion using this absolute position information will be investigated. 
Acknowledgements. The authors gratefully acknowledge the contributions of Simon Bachmann for assisting with the design and construction of the needle steering device, Simon DiMaio for providing advice and equipment for the experiments, Jesus Calvino-Fraga for helping in the development of the HC11 software, and Dave Fletcher for machining the parts of the steering device. This work was supported by the Natural Sciences and Engineering Research Council of Canada (NSERC).

\section{References}

1. Salcudean, S.E., Rohling, R.M., Okazawa, S.H., Ebrahimi, A.R., US Provisional Patent Application no. 60/435,263, Dec. 23, 2002.

2. DiMaio, S.P., Salcudean, S.E.: Needle Insertion Modeling for the Interactive Simulation of Percutaneous Procedures. MICCAI. (2002) 253-260

3. Personal communication. Morris, J. MD., B.C. Cancer Agency.

4. Smith, W.L., Surry, K.J.M., Mills, G.R., Downy, D.B., Fenster, A.: ThreeDimensional Ultrasound-Guided Core Needle Breast Biopsy. Ultrasound in Medicine and Biology. Volume 27(8) (2001) 1025-1034

5. Stoianovici, D., Whitcomb, L., Anderson, J., Taylor, R., Kavoussi, L.: A Modular Surgical Robotic System for Image Guided Percutaneous Procedures. Medical Image Computing and Computer-Assisted Intervention. (1998) 404-410

6. Cleary, K., Freedman, M., Clifford, M., Lindisch, D., Onda, S., Jiang, L.: ImageGuided Robotic Delivery System for Precise Placement of Therputic Agents. Journal of Controlled Release. Volume 74 (2001) 363-368

7. Patriciu, A., Solomon, S., Kavoussi, L., Stoianovici, D.: Robotic Kidney and Spine Percutaneous Procedures Using a New Laser-Based CT Registration Method. MICCAI. (2001) 249-257

8. Wunderbaldinger, P., Helbich, T.H., Partik, B., Turetschek, K., Wolf, G.: First Experience with a New Dedicated Ultrasound System for Computer-Guided LargeCore Breast Biopsy. European Radiology. Volume 11(12) (2001) 2460-2464

9. Loser, M.: A New Robotic System for Visually Controlled Percutaneous Interventions under X-ray or CT-Fluoroscopy. Dr. Ing. thesis. Univ. of Freiburg. 2002.

10. Daum, W.: Deflectable Needle Assembly. US Patent no. 5,572,593. June 3, 2003.

11. Ding, M., Cardinal, H., Guan, A., Fenster, A.: Automatic Needle Segmentation in 3D Ultrasound. In: Proceedings of SPIE: Medical Imaging. (2002) 23-28

12. Draper, K.J., Blake, C.C., Gowman, L., Downey, D.B., Fenster, A.: An Algorithm for Automatic Needle Localization in Ultrasound-Guided Breast Biopsies. Medical Physiscs. Volume 27(8) (2000) 1971-1979

13. Charboneau, J.W., Reading, C.C., Welch, T.J.: CT and Sonographically Guided Needle Biopsy: Current Techniques and New Innovations. American Journal of Roentgenology. Volume 154 (1990) 1-10

14. Powers, J.E.: Ultrasonic Imaging of Biopsy Needle. US Patent no. 5,095,910, March 17, 1992

15. Zhang, Y., Rohling, R., Pai, D.: Direct Surface Extraction from 3D Freehand Ultrasound Images. In: Proceedings of IEEE Visualization (2002) Oct 27 - Nov 1 